\title{
Generic degeneracy and entropy in loop quantum gravity
}

\author{
Mohammad H. Ansar:* \\ University of Waterloo, Waterloo, On, Canada N2L $3 G 1$ and \\ Perimeter Institute, Waterloo, On, Canada N2L $2 Y 5$
}

(Dated: September 17, 2018)

\begin{abstract}
Without imposing the trapping boundary conditions and only from within the very definition of area it is shown that the loop quantization of area manifests an unexpected degeneracy in area eigenvalues. This could lead to a deeper understanding of the microscopic description of a quantum black hole. If a certain number of semi-classically expected properties of black holes are imposed on a quantum surface its entropy coincides with the Bekenstein-Hawking entropy.
\end{abstract}

PACS numbers:

Contents

\section{Introduction}

\section{Generic degeneracy}

\section{Entropy \\ IV. Acknowledgement}

References

\section{INTRODUCTION}

In the early works on black holes in loop quantum gravity [1], it was first understood that the black hole entropy can be derived from the internal boundary of space without imposing any boundary condition. The underlying details of this pictures was later on recovered when the definition of a marginally trapped surface [2] was rewritten in the Ashtekar-Sen variables and this definition was extended into a quantum sector 3]. Such a surface after quantization contains a finite number of degrees of freedom and consequently carries an entropy that coincides with the Bekenstein-Hawking black hole entropy, [13]. In this note by the use of the same setting similar to the one of original works by Rovelli [1], we show that area operator acting on a typical inhomogeneous surface state manifests a degeneracy that here is worked out in both $\mathrm{SO}(3)$ and $\mathrm{SU}(2)$ group representations. This degeneracy exhibits a scale invariant correlation with area with the same exponent in both groups. However, this is not the only degeneracy an area eigenvalue is left with. Recently it was understood in [5] that the complete spectrum of area can be re-classified into different equidistant subsets. This symmetry (the so-called ladder symmetry) increases the total degeneracy such that the degeneracy of a large area eigenvalues becomes proportional to its area exponentially. Moreover, we present the derivation

*Electronic address: mansari@perimeterinstitute.ca of the Bekenstein-Hawking expression for the entropy of a Schwarzschild black hole of large surface area by the use of Dreyer's conjecture [6] that the minimal area of a hole in its dominant configuration should be identified with the emissive quanta from a perturbed black hole in highly damping mode. This motivates a rather different picture of a quantum horizon whose precise dynamical definition perhaps should be looked at from within a spin foam model, [7].

In loop quantization approach to quantum gravity the kinematical state space is taken to be $L^{2}$ (connections on SU(2) bundle). For any graph with finitely many edges and vertices embedded in a spatial manifold, the space of connections is $\mathrm{SU}(2)^{n}$ (or alternatively $\mathrm{SO}(3)^{n}$ ) where $n$ is the number of edges. In fact, a connection on a graph tells us how to parallel transport information along each edge of that graph. The canonical conjugate of this connection field represents the quantum geometry of space. The space of physical states is obtained by imposing constraints: the gauge-invariance, the diffeomorphism invariance, and the invariance under time evolution.

Given a graph and a surface in space, the area operator is supposed to be the quantum analog of the usual classical formula for the area of $S$. This operator only cares about the points where the graph intersects the surface. A subset of surface states which has no node residing on the surface was originally considered by Smolin and Rovelli where they derived their eigenvalues in [8]. Later on, all possible states of a surface were considered and the complete spectrum of area eigenvalues were found from different approaches, 9]. From the calculation, it was uncovered that those edges which are completely tangential to the surface do not contribute in the surface area, albeit the tangent vectors of crossing edges do contribute in it.

An edge with respect to an underlying surface falls into two classes. It may

- cross the surface at one intersecting point and bends at the surface on the point to induce a tangent vector on the surface, or

- reside completely tangential to the surface. 
A spin network with respect to three different surfaces $S_{1}, S_{2}$, and $S_{3}$ was shown in Figure (11). The quantum state of surface $S_{3}$ contains the bulk edges $j_{u}$ and $j_{d}$ and their tangent vector $j_{u+d}$ at their joint node. Note that the quantum states corresponding to the surfaces $S_{1}$ and $S_{2}$ contain the edges of equal spins on both sides without bending at these surfaces.

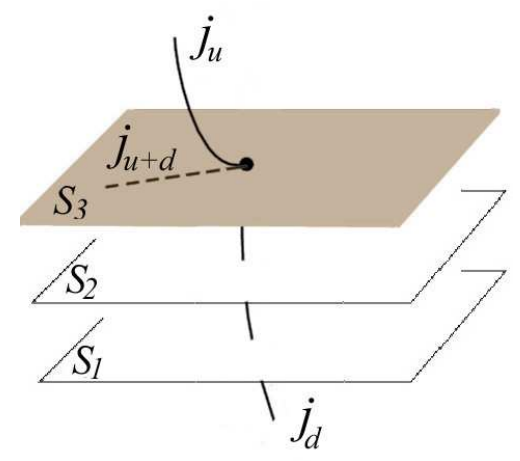

FIG. 1: The position of a surface relative to spin network.

The area eigenvalue associated with a typical quantum state $\left\langle j_{u}, j_{u+d}, j_{d}, j_{t}\right|$ is $a=m_{j_{u}, j_{u+d}, j_{d}} a_{o}$,

$$
m_{j_{u}, j_{u+d}, j_{d}}=\sqrt{2 f\left(j_{u}\right)+2 f\left(j_{d}\right)-f\left(j_{u+d}\right)},
$$

where $f(x)=x(x+1)$ and $a_{o}:=4 \pi \gamma \ell_{\mathrm{P}}^{2}$. The parameter $\gamma$ is a dimensionless parameter called the BarberoImmirzi parameter [10] and $\ell_{\mathrm{P}}$ is the Planck's length $\sqrt{\hbar G / c^{3}}$. Moreover, the tangent vector accept a finite number of quantum values from the following spectrum:

$$
j_{u+d} \in\left\{\left|j_{u}-j_{d}\right|,\left|j_{u}-j_{d}\right|+1, \ldots, j_{u}+j_{d}\right\} .
$$

The tangent vector at a node is a spin between the sum and difference of crossing edges at the node and resembles the total vector of two quantum angular momenta (i.e. spin and orbital angular momenta) in the Hydrogen atom. This pictorially is shown in Fig. (2) where the case (a) indicates $j_{u+d}=j_{u}+j_{d}$ and the case (c) indicates $j_{u+d}=\left|j_{u}-j_{d}\right|$. The case (b) in Figure (2) shows an intermediate value for the tangent vector between the maximum and minimum.

Let us now describe the subset of state that was reported first by Smolin and Rovelli as the area eigenstates in [8]. At a vertex if neither one of the upper and lower edges bends at the surface, the tangent vector becomes zero. In the lack of the completely tangential edges at the vertex, due to the gauge invariance, the upper and lower spins must be equal. The quantum of area in this case becomes $a=2 a_{o} \sqrt{j(j+1)}$. Therefore, the subset includes only those states with puncturing edges without bending at it.

Consider now a closed surface that divides space into two disjoint subsets of interior and exterior regions. Since

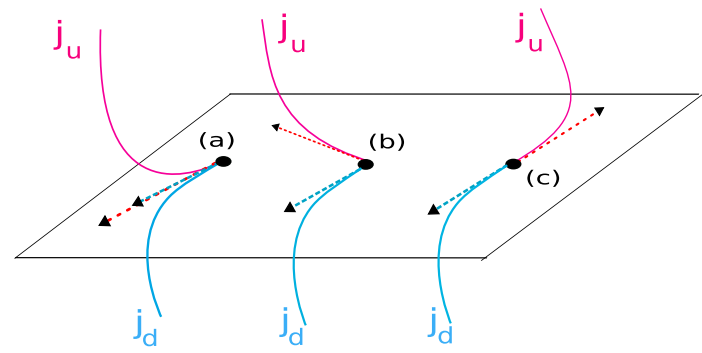

FIG. 2: Different components of the tangent vector of the upper and lower spins on the surface. The dashed arrows in blue and red colors corresponds to the edges in the lower and upper side

such a surface has no boundary a few additional vertices are needed in order to close its corresponding spin network. This makes unwanted contributions to the action of the area operator via the constraints:

$$
\sum_{\alpha} j_{u}^{(\alpha)} \in \mathrm{Z}^{+}, \quad \sum_{\alpha} j_{d}^{(\alpha)} \in \mathrm{Z}^{+},
$$

where $\alpha$ indicates the vertices of the graph. Note that these constraints are trivially satisfied in the $\mathrm{SO}(3)$ representation of the spin network states, because the spins are already in pairs (integer numbers). However, in $\mathrm{SU}(2)$ representation some spin network states are excluded by (3).

\section{GENERIC DEGENERACY}

For a given $j_{u}$ and $j_{d}$ a set of eigenvalues is generated from a minimum value (where $j_{u+d}=j_{u}+j_{d}$ ) to a maximum value (where $j_{u+d}=\left|j_{u}-j_{d}\right|$ ) by (11). Changing either $j_{u}$ or $j_{d}$, a different finite set of area eigenvalues is generated whose elements may or may not coincide with the elements of other set of area eigenvalues. Generating area eigenvalues from different eigenstates indicates that the spectrum of eigenvalues becomes denser in larger area. All of the eigenvalues are unexpectedly degenerate. In fact there exist a finite set of eigenstates that correspond to every area eigenvalue.

Let us consider the first four area eigenvalues in $\mathrm{SO}(3)$ group as samples. These are $a_{\min }=\sqrt{2} a_{o}, a_{2}=2 a_{o}$, $a_{3}=\sqrt{6} a_{o}$, and $a_{4}=2 \sqrt{2} a_{o}$, respectively. Note this $a_{4}$ is the minimal eigenvalue of the subset that was first discovered as the spectrum of area eigenvalues by integer spins. This area has been considered many times in the literature as the minimal area cell in the dominant configuration of a black hole horizon (i.g. see [1, 3, 4, 6]); however it is the double of the the minimal area eigenvalue $a_{\min }$ in the complete spectrum of (11).

The table (II) shows the detail of area states corresponding to these four area eigenvalues.

The three states corresponding to the minimal area are: 1) the state with the upper spin one edge crossing the surface and bending at it on the point. Such an edge 


\begin{tabular}{|c|c|c|c||c|c|c|c||c|c|c|c|}
\hline area & $j_{u}$ & $j_{u+d}$ & $j_{d}$ & area & $j_{u}$ & $j_{u+d}$ & $j_{d}$ & area & $j_{u}$ & $j_{u+d}$ & $j_{d}$ \\
\hline$a_{\min }$ & 0 & 1 & 1 & $a_{3}$ & 0 & 2 & 2 & $a_{4}$ & 1 & 0 & 1 \\
\hline$a_{\min }$ & 1 & 1 & 0 & $a_{3}$ & 1 & 1 & 1 & $a_{4}$ & 1 & 4 & 3 \\
\hline$a_{\min }$ & 1 & 2 & 1 & $a_{3}$ & 2 & 2 & 0 & $a_{4}$ & 3 & 4 & 1 \\
\hline$a_{2}$ & 2 & 4 & 2 & $a_{3}$ & 2 & 5 & 3 & $a_{4}$ & 3 & 7 & 4 \\
\hline$a_{2}$ & 2 & 3 & 1 & $a_{3}$ & 3 & 5 & 2 & $a_{4}$ & 4 & 7 & 3 \\
\hline$a_{2}$ & 1 & 3 & 2 & $a_{3}$ & 3 & 6 & 3 & $a_{4}$ & 4 & 8 & 4 \\
\hline
\end{tabular}

TABLE I: The eigenstates corresponding to the first four $\mathrm{SO}(3)$ eigenvalues.

induces all of its spin to the surface at the point. However a completely tangential edge is necessary to lie on the surface and ends at that point in order to make this state gauge invariant off the surface. 2) The state with lower spin one edge similar to the previous state. 3) The state with the upper and lower spins one edges bending at the surface such that their tangent vector come along each other in the same direction. This tangent vector could also connect to other completely tangential excitations on the surface.

In $\mathrm{SU}(2)$ group the eigenstates of the first six eigenvalues are tabulated in the table (III). These eigenvalues are: $a_{\min }=\frac{\sqrt{3}}{2} a_{o}, a_{2}=a_{o}, a_{3}=\frac{\sqrt{7}}{2} a_{o}, a_{4}=\sqrt{2} a_{o}$, $a_{5}=\frac{\sqrt{11}}{2} a_{o}$, and $a_{6}=\sqrt{3} a_{o}$. Note that the sixth eigenvalue is in fact the minimal area applied in the literature so far for the purpose of black hole entropy calculation in this group.

\begin{tabular}{|c|c|c|c||c|c|c|c||c|c|c|c|}
\hline area & $j_{u}$ & $j_{u+d}$ & $j_{d}$ & area & $j_{u}$ & $j_{u+d}$ & $j_{d}$ & area & $j_{u}$ & $j_{u+d}$ & $j_{d}$ \\
\hline$a_{\min }$ & 0 & $\frac{1}{2}$ & $\frac{1}{2}$ & $a_{4}$ & 0 & 1 & 1 & $a_{6}$ & $\frac{1}{2}$ & 0 & $\frac{1}{2}$ \\
\hline$a_{\min }$ & $\frac{1}{2}$ & $\frac{1}{2}$ & 0 & $a_{4}$ & 1 & 1 & 0 & $a_{6}$ & $\frac{1}{2}$ & 2 & $\frac{3}{2}$ \\
\hline$a_{2}$ & $\frac{1}{2}$ & 1 & $\frac{1}{2}$ & $a_{4}$ & 1 & 2 & 1 & $a_{6}$ & $\frac{3}{2}$ & 2 & $\frac{1}{2}$ \\
\hline$a_{3}$ & $\frac{1}{2}$ & $\frac{3}{2}$ & 1 & $a_{5}$ & 1 & $\frac{5}{2}$ & $\frac{3}{2}$ & $a_{6}$ & $\frac{3}{2}$ & 3 & $\frac{3}{2}$ \\
\hline$a_{3}$ & 1 & $\frac{3}{2}$ & $\frac{3}{2}$ & $a_{5}$ & $\frac{3}{2}$ & $\frac{5}{2}$ & 1 & & & & \\
\hline
\end{tabular}

TABLE II: The eigenstates corresponding to the first six $\mathrm{SU}(2)$-valued spin networks.

The minimal area in this group is degenerate in the two states each with a crossing spin one-half edge bending at the surface. In these states there must be at least one completely tangential edges of spin one-half connecting to the intersecting point.

Note that in the degenerate eigenvalues we investigated here, which are the first a hundred levels, there is only one state that exceptionally does not appear as a degenerate state and that is the state with area $a_{2}$ in $\mathrm{SU}(2)$ group. In this state the upper and lower edges of spins one-half connect at a vertex and bend at the surface along the same direction.

We counted the number of the degeneracy $g$ for different eigenvalues in scatterplots and the area and its degeneracy appeared to be correlated in Figure 3 These scatterplots indicates the results in $\mathrm{SU}(2)$ and $\mathrm{SO}(3)$ group representations of spin networks separately in the log-log graphs.

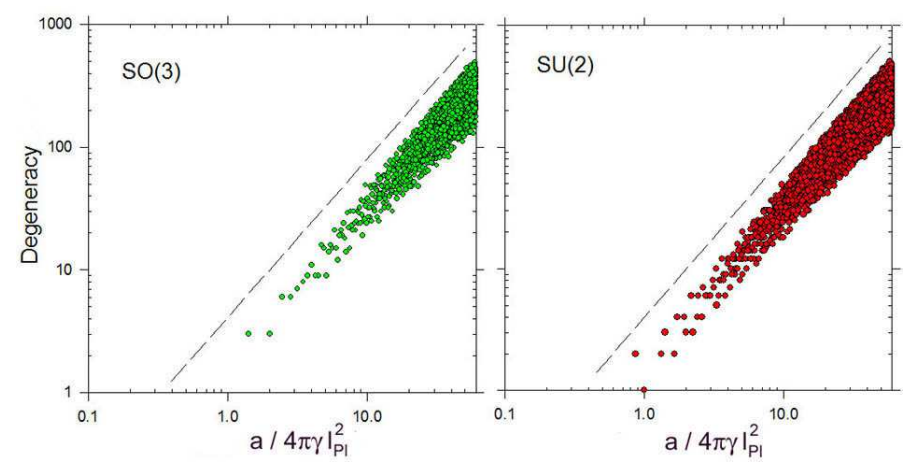

FIG. 3: The scatterplot of correlation between the area eigenvalues and the degeneracies of their corresponding eigenstates.

The eigenvalue degeneracy grows roughly as a powerlaw but with increasing scatter, namely

$$
g(a) \approx\left(\frac{a}{a_{o}}\right)^{\alpha+\epsilon}
$$

where $\alpha=0.96$ with the scatter uncertainty $\epsilon= \pm 0.03$. This particular degeneracy behaves scale invariant and is robust, however we will so in this note later on that this is not the only degeneracy of eigenvalues.

For the purpose of further clarifications, we plot the minimal area levels and their corresponding degeneracy separately in Figure (4).

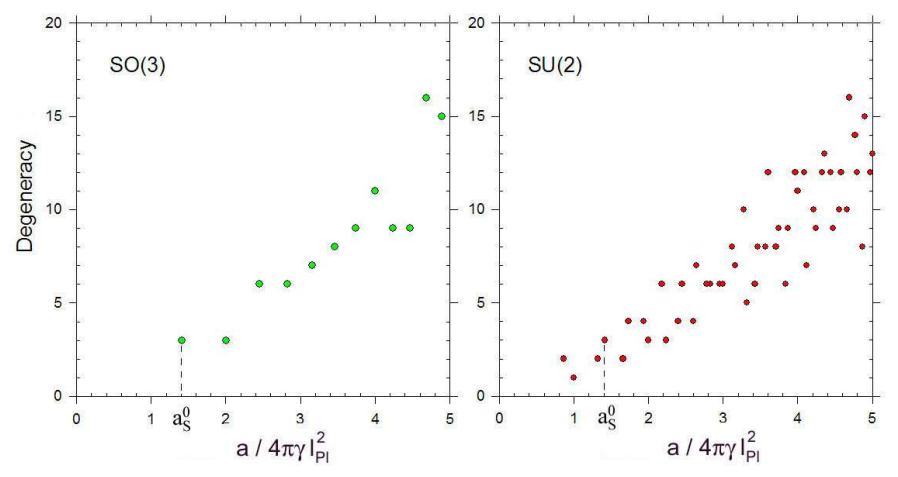

FIG. 4: The scatterplot of a few first area level degeneracies.

In fact it is obvious that we do not need two different plots for the $\mathrm{SO}(3)$ and $\mathrm{SU}(2)$ groups because the spectrum of area in the $\mathrm{SO}(3)$ group is contained in the $\mathrm{SU}(2)$ group degeneracy graph. For instance, in Figure (4) we named the minimal area in the $\mathrm{SO}(3)$ group by $a_{o}^{S}$ and showed this area is the fourth level in the $\mathrm{SU}(2)$ group. More importantly this is the reason why the exponent of the power law (44) in the both group representation is the same.

Recently the complete spectrum of area is shown to be the union of equidistant spectra, [5]. Each one of the subsets possesses a gap between levels equal to $a_{o} \chi \sqrt{\zeta}$. In 
$\mathrm{SO}(3)$ group $\zeta$ is any square-free number $\{1,2,3,5, \cdots\}$ and the group characteristic parameter is $\chi:=\sqrt{2}$; and in $\mathrm{SU}(2)$ group $\zeta$ is the discriminant of any positive definite form $\{3,4,7,8,11, \cdots\}$ and the group characteristic parameter is $\chi:=1 / 2$. In other words, the complete spectrum reformulates into $a_{n}(\zeta)=a_{0} \chi \sqrt{\zeta} n$ for $\forall n \in \mathbb{N}$. Fixing $\zeta$ a generation of evenly spaced numbers is singled out. The parameter $\zeta$ is therefore called the generational number.

Based on this classification, one can re-classify the generic degeneracy of area levels into the generations. Plots (a-e) in Figure (5) indicates this classification in the first five generation. Plot (f) compares the degeneracy of the first level of different generations.
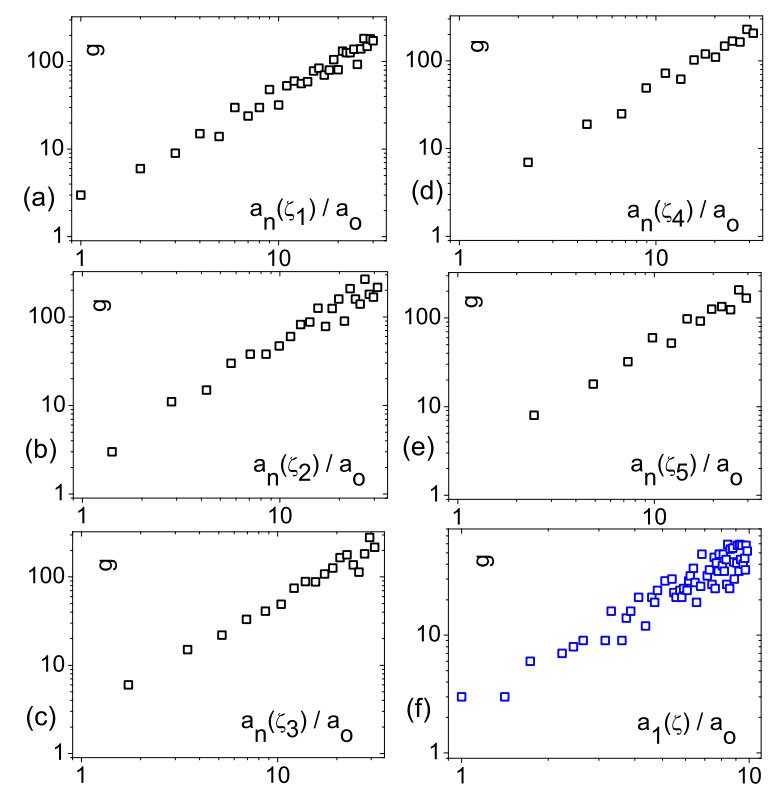

FIG. 5: The generic degeneracy of figure (3) classified into generation in $\mathrm{SO}(3)$ group case.

Note that in any generation the area of higher levels can be decomposed precisely into smaller fractions of the same generation (without approximation) namely, $a_{n}=n a_{1}=(n-2) a_{1}+a_{2}=\cdots$. Let us consider for instance the configuration $n a_{1}$. Each one of these area cells is degenerate. However, the states corresponding to the eigenvalue in different regions of the surface are distinguishable because by definition various number of completely tangential edges with various spins could be connected to each vertex without changing the area and the geometric configurations. Therefore, the degeneracy of the area eigenvalue $a_{n}$ is in fact $\Omega_{n}=g_{n}+g_{n-1} g_{1}+\cdots+\left(g_{1}\right)^{n}$. Obviously the dominant term in the sum belongs to the configuration with maximum number of the area cell $a_{1}$.

\section{ENTROPY}

Consider a surface $S$ of a large area $A$. This area is the sum of quanta in different configurations. In the dominant configuration it contains the maximum number $(\mathrm{N})$ of minimal degenerate area cell; $A \approx N a_{\text {min }}$, where $N \gg 1$. Let us now consider the surface is a black hole horizon. By the use of Einstein equation for a collapsing star from a non-spherical state all radiatable perturbations to the surface is radiated away such that at the late stage the hole is left only with its monopoles. Now imagine the initial deformation is located in a certain region of the horizon. The future evolution of the field from that point depends on the exact spin network state of that location, which includes the states of completely tangential excitations. The state evolves under the action of a Hamiltonian and is expected to radiate away energy from the event horizon. Therefore, in the quantum states of a black hole the complete information of spin network states makes regions distinguishable from each other, although they may appear with the same area. Having defined the dominant configuration for the surface, the degeneracy of this configuration is therefore $\Omega(A)=g\left(a_{\min }\right)^{N}$. Consequently, the dominant entropy associated to the underlying surface is proportional to $N \ln g\left(a_{\min }\right)$ or equivalently

$$
S=A \ln g\left(a_{\min }\right) / a_{\min } .
$$

It is instructive to compare this result with the method the same entropy is derived from the boundary picture. In that picture the hole separates the space manifold into two sections, namely a horizon boundary and the outer space. Gauge degrees of freedom are still considered to be redundant in the bulk states but they become physical degrees on the boundary. The reason is that the kinematical Hilbert space of space includes the Hilbert spaces of the horizon $\mathcal{H}_{s}$ and the bulk $\mathcal{H}_{b}$. The reduction of gauge degrees of freedom from this space takes place only in the bulk partition because $\left\{\mathcal{H}_{s} \otimes \mathcal{H}_{b}\right\} / S U(2) \sim$ $\mathcal{H}_{s} \otimes\left\{\mathcal{H}_{b} / S U(2)\right\}$. This means the horizon Hilbert space accepts the gauge transformation redundancies as the physical states. An edge of spin $j$ puncturing the boundary produces $2 j+1$ physical states on the surface. By assuming that different area cells on the horizon are distinguishable the dominant configuration is the one with a maximum number of the minimal area $a_{\mathrm{min}}^{\text {(punc) }}$ (the area of spin $j_{\text {min }}$ puncture). Consequently, black hole entropy becomes $S=A \ln \left(2 j_{\min }+1\right) / a_{\min }^{\text {(punc) }}$. Note that, as it was mentioned above, the minimal quantum of area in this calculation is not the minimal area in the complete spectrum of area. Now the question is that based on what reason this subset was first considered as the basis for representing the geometry of a horizon? The easy answer to this is the one has been mentioned by Rovelli in his original work [1] that none of the other eigenvalues was known at the time the black hole entropy was studied. However, there is a more sophisticated and physical 
answer to this. As soon as one assumes a black hole horizon as the internal boundary of space, the contribution of the boundary to the gravity action of the space becomes the Chern-Simons action, 14]. When this space is quantized the geometry of this boundary, in principle, is described by a set (and not a sequence) of punctures. As it was mentioned above, the gauge degrees of freedom on this boundary become physical. Regardless of the group representation that suits the boundary fields (which could be either $\mathrm{SU}(2)$ or $\mathrm{U}(1)$ ), since the internal spin network states in this approach are removed out, the boundary is left only with a subset quanta (those which puncture the boundary).

However, beyond postulations and assumptions there is no physical reason to outlaw considering the spin network states in the interior region of black hole. Indeed, the black hole interior may be in an infinite number of states. For instance, the black hole interior may be given by a Kruskal extension so that on the other side of the hole there is another universe. The inclusion of these states in fact allows the horizon to be quantized via the complete spectrum of area. However, the number of those internal states cannot affect the interaction of the hole with its surroundings. From the exterior, the hole is completely determined by the properties of its surface. Thus, the entropy relevant for the thermodynamical description of the thermal interaction of the hole with its surroundings is determined by the states of the quantum gravitational field on the black hole surface.

In principle, depending on the Hamiltonian operator that maps the physical Hilbert space of the interior region of a closed surface into itself, the surface may evolve and its area may increase, decrease or become unchanged in the course of time. In the case of black hole, the time evolution map is responsible for its non-decreasing area character and turning the kinematic entropy we reported here into a physical non-decreasing entropy, [11]. This motivates a picture of a more quantum black hole in which the quantization of space occurs prior to the definition of the black hole sector. Here we restrict the results into only the kinematical ones and disregard arguing about the dynamics. Of course one way to start the definition of such a horizon perhaps is possible through a spin foam model, (for review of different models see [7]).

A non-rotating spherical black hole 'responses' any perturbation by some complex frequencies called quasinormal modes. This makes a black hole horizon state different from a random surface. The imaginary part of the modes is the frequency at which the perturbation is damped. The real part is the frequency at which a quantum of energy is emitted from the black hole or is absorbed into it. When the perturbation is damped very quickly, the response of the black hole are the emission of the quantum of energy $\Delta M=M_{\mathrm{Pl}}^{2} \ln 3 / 8 \pi M$, [12]. Since the quanta of energy and area are proportional to each other $\Delta M=\Delta A\left(c^{4} / 32 \pi G^{2} M\right)$, the exchanging area from the black hole is $\Delta A=4 \ln 3 \ell_{\mathrm{P}}^{2}$.

Following Dreyer's conjecture [6] for identifying the classical perturbation of a black hole horizon in highly damping mode with the transition between the two natural configurations, it can be assumed $\Delta A=a_{\text {min }}$. From this equivalence the entropy of a black hole in Planck's area unit becomes

$$
S=A \ln g_{1}\left(\zeta_{\min }\right) / 4 \ln 3
$$

in any group representation.

Unexpectedly in $\mathrm{SO}(3)$ group, since the degeneracy of the minimal area is three, the two logarithms from the numerator and denominator of (6) are canceled out and the Bekenstein-Hawking entropy is verified. This lets us to calculate the Barbero-Immirzi parameter by the size of minimal area. This minimal area in this picture is a half times smaller than the minimal area on a boundary. The reason is that here we do not restrict the horizon to be a boundary of space and the internal spin network exists and connects to the horizon surface. The Barbero-Immirzi becomes tuned here to the value $\gamma=\frac{\ln 3}{\pi \sqrt{2}}$. This value is the double of what has been reported using Dreyer's result in a boundary picture of horizon.

In summary, we reported that the complete spectrum of area possesses eigenvalue degeneracy. This degeneracy with respect to area in both group representations is power law with increasing scatter. However since the complete spectrum of area is the union of different equidistant subsets, the total degeneracy of a large eigenvalue becomes proportional exponentially to its area. The black hole entropy relevant for the thermodynamical interaction of a black hole with its exterior region is the number of the quantum microstates of the horizon which are distinguishable from the exterior of the hole. We have obtained that the entropy is proportional to the area. Moreover we derived the exact form of the Bekenstein-Hawking formula when the minimal area is considered to be the quantum of area emitted from the hole in its highly damping mode.

\section{ACKNOWLEDGEMENT}

Helpful discussions with K. Krasnov, C. Rovelli, L. Smolin, and R. Sorkin are acknowledged.
[1] "Black Hole Entropy from Loop Quantum Gravity", Physical Review Letter 14, 3288 (1996); "Loop Quan- tum Gravity and Black Hole Physics", Helvetica Phys- 
ica Acta, 69 (1996) 582; gr-qc/9608032 "Loop Quantum Gravity" by Carlo Rovelli, Cambridge University Press 2005.

[2] B. K. Berger, D. M. Chitre, Y. Nutku, V. E. Moncrief, Phys. Rev. D5, 2467-2470 (1971); S. W. Hawking, Commun. Math. Phys. 43 (1975) 199; W. Unruh, Phys. Rev. D14, 870 (1973); P. Hajicek, Phys. Rev. D30 1178 (1984); T. Thiemann and H. A. Kastrup, Nucl. Phys. B425, 665 (1994); K. Kuchar, Phys. Rev. D50, 3961 (1994).

[3] A. Ashtekar, J. Baez, A. Corichi and K. Krasnov, "Quantum geometry and black hole entropy," Phys. Rev. Lett. 80, 904 (1998) arXiv:gr-qc/9710007. ; A. Ashtekar, J. C. Baez and K. Krasnov, "Quantum geometry of isolated horizons and black hole entropy," Adv. Theor. Math. Phys. 4, 1 (2000) arXiv:gr-qc/0005126 ; L. Smolin, "Linking topological quantum field theory and nonperturbative quantum gravity," J. Math. Phys. 36 (1995) 6417. arXiv:gr-qc/9505028; K. V. Krasnov, "Counting surface states in the loop quantum gravity," Phys. Rev. D 55, 3505 (1997) arXiv:gr-qc/9603025; K. V. Krasnov, "On statistical mechanics of gravitational systems," Gen. Rel. Grav. 30, 53 (1998) arXiv:gr-qc/9605047.

[4] E. Livine, D. Terno, "Quantum black holes: Entropy and entanglement on the horizon," arXiv:gr-qc/0508085. E. Livine, D. Terno, "Reconstructing quantum geometry from quantum information: Area renormalisation, coarse-graining and entanglement on spin networks," arXiv:gr-qc/0603008 D. Terno, "From qubits to black holes: Entropy, entanglement and all that," Int. J. Mod. Phys. D 14, 2307 (2005).

[5] M. H. Ansari, "Spectroscopy of a canonically quantized horizon," Nucl. Phys. B 783, 179 (2007) arXiv:hep-th/0607081.

[6] O. Dreyer, "Quasinormal modes, the area spectrum, and black hole entropy," Phys. Rev. Lett. 90, 081301 (2003). arXiv:gr-qc/0211076.
[7] A. Perez, "Spin foam models for quantum gravity," Class. Quant. Grav. 20, R43 (2003) arXiv:gr-qc/0301113.

[8] C. Rovelli and L. Smolin, "Discreteness of area and volume in quantum gravity," Nucl. Phys. B 442, 593 (1995) [Erratum-ibid. B 456, 753 (1995)]. arXiv:gr-qc/9411005;

[9] A. Ashtekar and J. Lewandowski, "Quantum theory of geometry. I: Area operators," Class. Quant. Grav. 14, A55 (1997). arXiv:gr-qc/9602046; S. Frittelli, L. Lehner and C. Rovelli, "The complete spectrum of the area from recoupling theory in loop quantum gravity," Class. Quant. Grav. 13, 2921 (1996) arXiv:gr-qc/9608043.

[10] J. F. Barbero, "Real Ashtekar variables for Lorentzian signature space times," Phys. Rev. D 51, 5507 (1995) arXiv:gr-qc/9410014; G. Immirzi, "Real and complex connections for canonical gravity," Class. Quant. Grav. 14, L177 (1997) arXiv:gr-qc/9612030.

[11] R. Sorkin, Stud. Hist. Philos. Mod. Phys. 36, 291 (2005); R. Wald, Living Rev. Rel. 4, 6 (2001).

[12] H. P. Nollert, "TOPICAL REVIEW: Quasinormal modes: the characteristic 'sound' of black holes and neutron stars," Class. Quant. Grav. 16 (1999) R159; K. D. Kokkotas and B. G. Schmidt, "Quasi-normal modes of stars and black holes," Living Rev. Rel. 2 (1999) 2 arXiv:gr-qc/9909058; S. Hod, "Bohr's correspondence principle and the area spectrum of quantum black holes," Phys. Rev. Lett. 81 (1998) 4293 arXiv:gr-qc/9812002; A. Alekseev, A. P. Polychronakos and M. Smedback, "On area and entropy of a black hole," Phys. Lett. B 574, 296 (2003) arXiv:hep-th/0004036.

[13] Recently a different approach towards this entropy from the use of quantum information theory techniques in loop quantum gravity has also been developed in [4].

[14] This is also true in any BF theory action. More precisely nothing enters into this surface term from the special character of gravity as a constrained BF theory. 